\title{
The lateral intercostal artery perforator as an alternative donor vessel for free vascularized lymph node transplantation
}

\author{
Min-Seok Daniel Kwak, Hans-Guenther Machens \\ Department of Plastic Surgery and Hand Surgery, Klinikum rechts der Isar, Technical University of Munich, Munich, Germany
}

Chronic lymphedema is caused by an impairment of the lymphatic system due to primary or secondary causes. Vascularized lymph node transplantation (VLNT) is currently the most promising and frequently used technique besides lymphaticovenous anastomosis. However, the vessel anatomy in the lateral thoracic region is sometimes quite variable. Based on our experiences with vascular anatomical inconstancy in the lateral thoracic region, we planned a lateral intercostal artery perforator flap for VLNT in a female patient with chronic stage II lymphedema of both legs after cervical cancer treatment. After surgery, the patient reported significant improvement in limb volume and the accompanying symptoms. The limb circumference was reduced by an average of 19.2\% at 6 months postoperatively. Despite having a short pedicle and small vessel caliber, the lateral intercostal artery perforator flap can safely be used for VLNT in lymphedema patients with anatomical variants.

Keywords Lymphedema / Transplantation, autologous / Perforator flap / Microsurgery / Free tissue flaps
Correspondence: Min-Seok Daniel Kwak Department of Plastic Surgery and Hand Surgery, Klinikum rechts der Isar, Technical University of Munich, Ismaninger Strasse 22, 81675 Munich, Germany

Tel: +49-89-4140-2171

Fax: +49-89-4140-4869

E-mail:min-seok.kwak@mri.tum.de

The authors would like to thank Haydar Kuekrek for surgical assistance.

\section{INTRODUCTION}

Vascularized lymph node transplantation (VLNT) was first pioneered by Becker et al. [1]. They treated 24 female patients with secondary chronic arm lymphedema after breast cancer treatment with good results. One of the main drawbacks of VLNT is iatrogenic lymphedema at the donor site, especially in the arm and leg region [2]. For this reason, further options for lymph node harvest have been developed [3-6].

Mardonado et al. [3] presented a prospective study with 100 consecutive cases of supraclavicular lymph node flaps. They reported no flap loss and no cases of iatrogenic lymphedema at the donor site. In 2017, Nguyen et al. [5] presented 42 patients who underwent a free omental lymphatic flap with significant symptom improvement. A substantial benefit of omental lymphatic flaps is avoiding the possibility of iatrogenic lymphede$\mathrm{ma}$, but a double-boarded plastic and general surgeon is necessary to harvest the flap. Finally, Poccia et al. [7] described a platysma-sparing submental lymph node flap that did not cause donor site lymphedema.

Since February 2011, we have treated 78 chronic lymphedema patients with VLNT. Our usual donor sites are the groin and the lateral thoracic region. In some cases, we have experienced very inconsistent vessel anatomy in the lateral thoracic region. Usually, our lymph node flaps are based on a side branch of the thoracodorsal or lateral thoracic artery, but sometimes those vessels 
are difficult to find due to extensive anatomic variance. In such cases, the lateral intercostal artery perforator (LICAP) is a reliable alternative as a donor vessel.

\section{CASE}

This study was authorized by the ethical review committee (IRB No. 7/18s).

The patient was a 35-year-old woman (height, $1.67 \mathrm{~m}$; weight, $85 \mathrm{~kg}$; body mass index, $30.5 \mathrm{~kg} / \mathrm{m}^{2}$ ) who had suffered from chronic stage II lymphedema of both lower extremities, including both feet, after cervical cancer therapy (lymphadenectomy, irradiation, and chemotherapy) since 2015. The lymphedema was more distinctive on the right side. The accompanying symptoms were chronic pain, meteoropathy, skin tension, and reduced mobility. She irregularly received manual lymph drainage (MLD), wore compression garments, and also suffered from hypothyroidism. She regularly took diuretics and L-thyroxine. She was also an active smoker (29.4 pack-years) and did not quit perioperatively (Fig. 1).

We use specific self-developed preoperative, intraoperative, and postoperative questionnaires as standardized documentation, which include information about the recipient and donor sites. Prior to surgery, lymphoscintigraphy of both lower limbs was performed, revealing delayed lymph drainage on the right side. After 7 minutes, only the left groin lymph nodes could be visualized. Limb circumference was measured manually from the distal to proximal aspects at the levels of $4 \mathrm{~cm}, 20 \mathrm{~cm}$, and 40 $\mathrm{cm}$ using a measuring tape (starting from the ankle). The perfo-

Fig. 1. Chronic stage II lymphedema in lower extremities

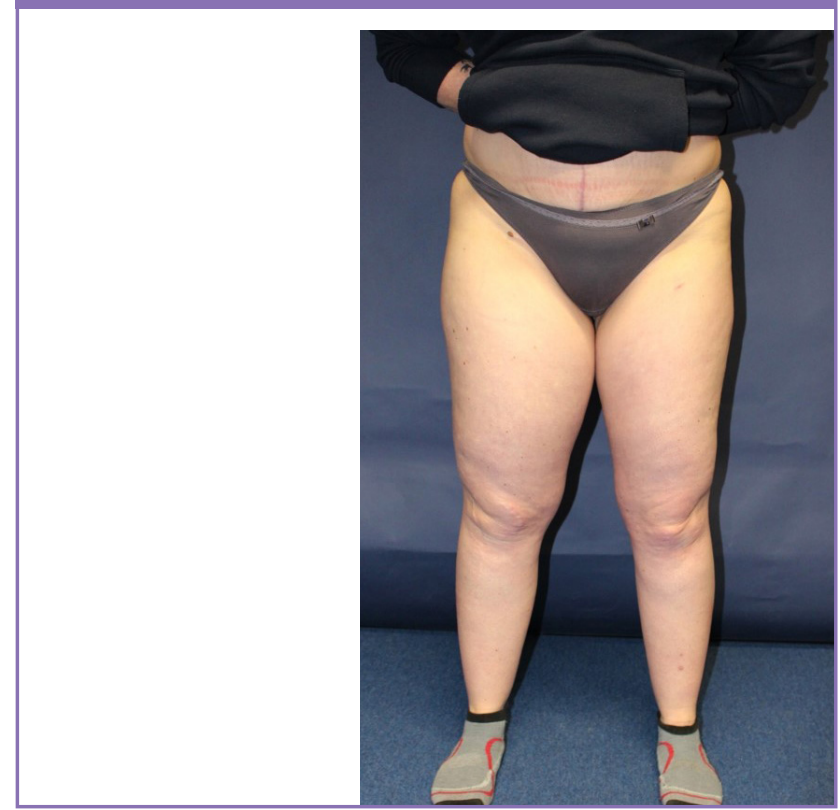

rators were marked in the left thoracic region using Doppler ultrasonography. After obtaining written informed consent, 2 teams participated in the procedure (Fig. 2).

One team harvested the lymph node flap from the left thoracic region, while the other team prepared the recipient site in the right groin region for implantation. Loupe magnification was used during preparation. The femoral artery and vein with their side branches were exposed and scar tissue was removed meticulously. After a skin incision, the skin was detached from the flap (fat tissue), which was located at the same level as the nipple on the anterior axillary line (level 1). A higher preparation was avoided to prevent lymphedema formation. The flap was then harvested from the distal to proximal aspects, and the muscle fascia from the thorax was included. Two LICAPs could be found nourishing the flap. Both perforators and their concomitant veins were isolated and dissected all the way to the intercostal artery. To avoid the risk of lung injury (e.g., pneumothorax), the dissection was ended here. The larger-caliber artery (around $0.4-0.5 \mathrm{~mm}$ ) was chosen as the donor vessel and the flap was prepared for microanastomosis using a microscope. The flap size was approximately $9 \times 10 \mathrm{~cm}$ (Fig. 3).

The recipient vessels were side branches from the femoral artery and vein, and anastomoses were performed using Ethilon sutures (10-0 sutures for the artery and 11-0 for the vein). Afterwards, the flap was placed over the main vessels and fixed subcutaneously with Vicryl 4-0 sutures. Drains were placed in both wounds and closed using multilayered Vicryl (3-0 and 4-0) and Monocryl (3-0) sutures. The patient stayed in the hospital for 7 days, and received $500 \mathrm{mg}$ of oral cefuroxime 3 times per day for

Fig. 2. Preoperative lymphoscintigraphy 7 minutes after the injection 


\section{Fig. 3. Surgical approach for harvesting}

(A) Preoperative markings of the skin incision, flap size, and perforators. (B) Two perforators from the left intercostal artery (LICAP) were identified during the dissection. (C) LICAP lymph node flap after harvesting, with the vein and artery clip-marked. LICAP, lateral intercostal artery perforator.
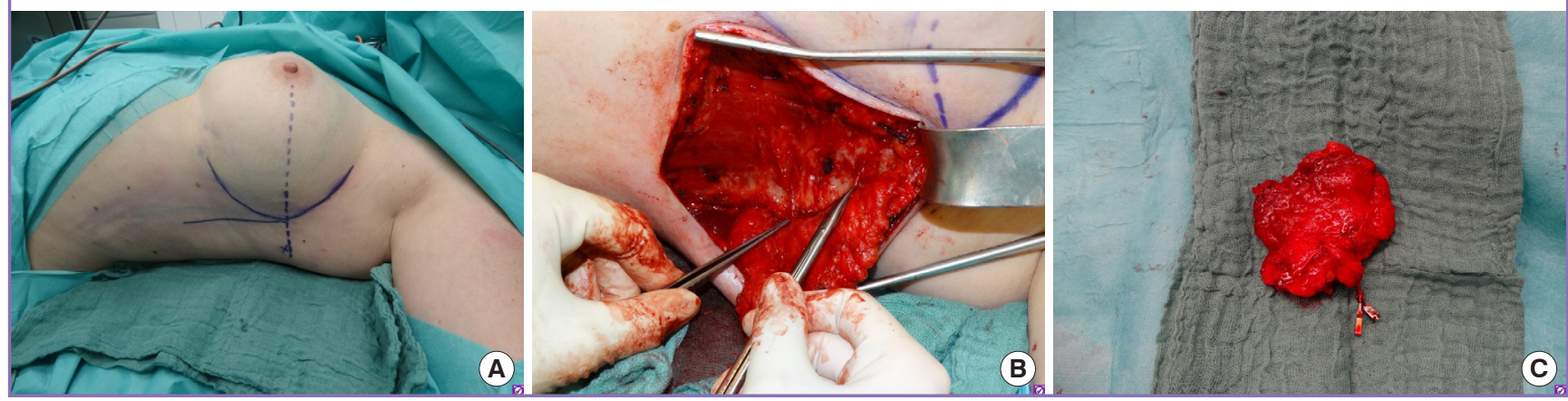

\section{Fig. 4. Six weeks after surgery}

Six weeks after vascularized lymph node transplantation with a lateral intercostal artery perforator flap.

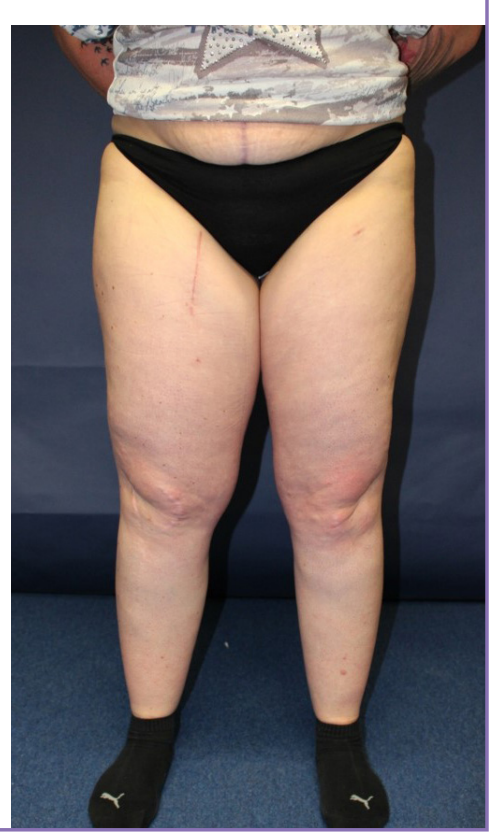

1 week and prophylactic anticoagulation subcutaneously. Starting 1 day after surgery, she was allowed to mobilize herself, without flexing her hip for 4 weeks. MLD was started immediately, without involving the surgical site for 4 weeks. After 4 weeks, full MLD was permitted. The patient came to the outpatient department for follow-up examinations at 6 weeks and 6 months postoperatively. At each appointment, photos were taken, the limb circumference was measured, and the patient's history was documented using our questionnaire.

Six weeks postoperatively, all wounds had healed without any complications. No signs of infection or implant failure could be detected. The mean reduction of limb circumference was $26.8 \%$ (Fig. 4).

After 6 months, the patient reported no pain, meteoropathy, or skin tension, and her mobility had improved. The tissue was soft-
Fig. 5. Postoperative lymphoscintigraphy 10 minutes after the injection

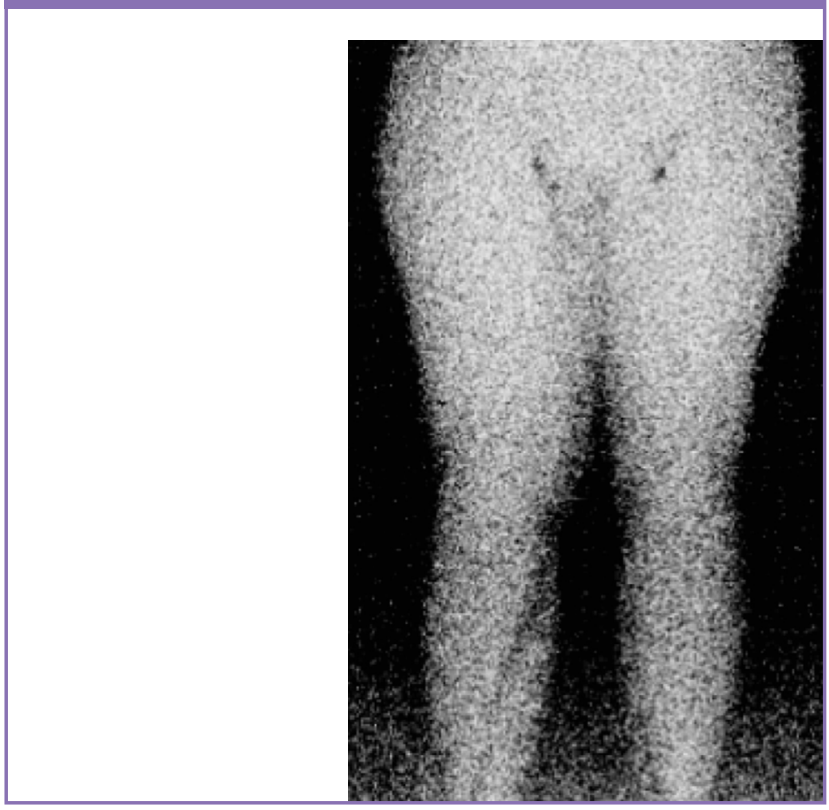

er and she reported improvement in the volume of edema. The 6-month evaluation indicated that she did not need to receive complex decongestive therapy, and the mean reduction of the limb circumference was found to be $19.2 \%$. Lymphoscintigraphy at 6 months showed a significant improvement in lymph drainage on the right side. After 10 minutes, the groin lymph nodes on both sides could be detected and the lymph drainage was symmetric in comparison to the preoperative imaging (Fig. 5).

For upcoming follow-ups (at $1,1.5$, and 2 years) the patient's history and limb circumference will be documented, and photos will be taken again. After 1 year, lymphoscintigraphy will be performed again. If there is no significant improvement after 1 or 2 years, a 2 nd lymph node transplantation into the popliteal space or ankle region can be considered. Alternatively, lymphaticovenous anastomoses can be performed (Tables 1, 2 and Fig. 6). 
Table 1. Clinical results after 6 months

\begin{tabular}{|lll|}
\hline Clinical data & $\begin{array}{c}\text { Preoperative } \\
\text { status }\end{array}$ & $\begin{array}{c}\text { Postoperative } \\
\text { status }\end{array}$ \\
\hline Pain & Yes & No \\
Skin tension & Yes & No \\
Meteoropathy & Yes & No \\
Reduced mobility & Yes & Improvement \\
Compression garments & Yes & No \\
Complex decongestive therapy & Irregular & No \\
Lymphoscintigraphy & Impaired & Improved uptake \\
\hline
\end{tabular}

\section{Fig. 6. Six months after surgery}

The patient reported no pain, skin tension, or meteoropathy, and her mobility had improved.

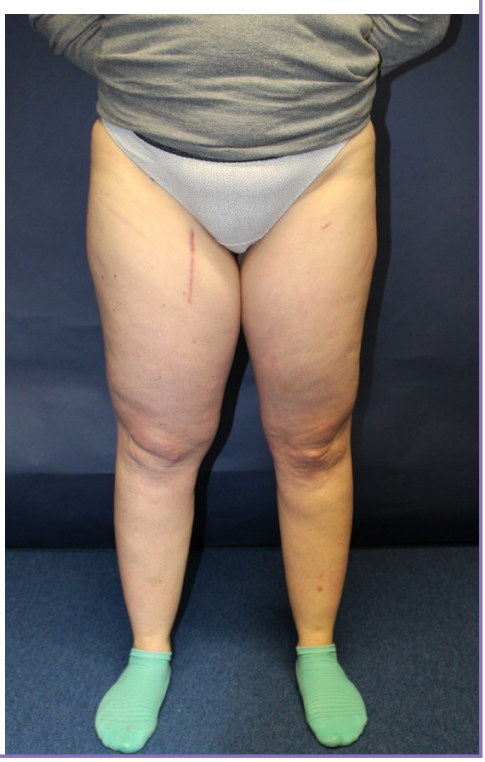

\section{DISCUSSION}

The field of surgical lymphedema treatment is still improving, and there is no generally recognized state-of-the-art technique [8]. Currently, VLNT and lymphaticovenous anastomoses are the most promising approaches to treat chronic lymphedema $[9,10]$. Increasingly, many reconstructive surgeons tend to combine these 2 techniques to enhance their results [11]. However, the correct indications must be considered. Lymphatic surgery in general is quite sophisticated in terms of the required technical skills and experience in microsurgery. Moreover, the evolution of high-quality microinstruments has contributed to the rapid improvements in microsurgery.

To minimize iatrogenic lymphedema formation at the donor site, a supraclavicular, submental, or omental lymphatic free flap should be considered, and these techniques should be included in the repertoire of a reconstructive microsurgeon. Except for the quite long learning curve for harvesting a supraclavicular or submental lymphatic free flap and the need for a general sur-
Table 2. Improvement of limb circumference after LICAP flap

\begin{tabular}{|c|c|c|c|}
\hline \multirow{2}{*}{$\begin{array}{l}\text { Measurement } \\
\text { level }\end{array}$} & \multirow{2}{*}{$\begin{array}{l}\text { Preoperative CF } \\
\text { (cm) }\end{array}$} & \multicolumn{2}{|c|}{ Postoperative CF } \\
\hline & & $\begin{array}{l}6 \text { Weeks } \\
(\mathrm{cm})\end{array}$ & $\begin{array}{l}6 \text { Months } \\
\text { (cm) }\end{array}$ \\
\hline $4 \mathrm{~cm}$ & 32 & $23.0(-28.1)$ & $26.0(-18.8)$ \\
\hline $20 \mathrm{~cm}$ & 48 & $40.4(-15.8)$ & $41.5(-13.5)$ \\
\hline $40 \mathrm{~cm}$ & 73 & $46.4(-36.4)$ & $56.0(-23.3)$ \\
\hline Mean circumference & 51 & $36.6(-26.8)$ & $41.2(-19.2)$ \\
\hline
\end{tabular}

geon to harvest an omental lymphatic free flap, all these procedures seem to be reproducible and safe in the hands of a skilled and experienced plastic surgeon.

However, the LICAP flap as a free vascularized lymph node transplant from the lateral thoracic region is a good alternative option if the usual donor vessels, such as the thoracodorsal or lateral thoracic artery, are lacking or show considerable anatomic variance. The LICAP flap was previously described as a free flap, but is generally considered a pedicled flap by various authors, and its use has been reported for different indications [1215]. Hwang et al. [12] also experienced vessel variance in the lateral thoracic region and therefore switched to the LICAP flap. Especially for demanding breast reconstruction procedures, the local LICAP flap provides additional opportunities [15]. In recent years, a sensate intercostal artery perforator flap for plantar reconstruction was even presented by lida et al. [14] in 2014.

Unlike recent authors, we used a free LICAP lymph node flap for the treatment of chronic lymphedema. The vessel anatomy of LICAPs is constant and the dissection procedure is quite simple. Despite the short pedicle and the small vessel size, the LICAP flap can be anastomosed safely in the recipient region. Careful dissection should be performed near the main vessel (intercostal artery) to avoid thoracic injuries such as pneumothorax. Additionally, the intercostal nerve should be preserved to prevent postoperative pain or discomfort in the thoracic region. The LICAP flap is a reliable alternative if there are no other side branches or perforators during VLNT.

\section{NOTES}

\section{Conflict of interest}

No potential conflict of interest relevant to this article was reported.

\section{Ethical approval}

The study was approved by the Institutional Review Board of 
Technical University of Munich (IRB No. 7/18s) and performed in accordance with the principles of the Declaration of Helsinki. Written informed consents were obtained.

\section{Patient consent}

The patient provided written informed consent for the publication and the use of her images.

\section{REFERENCES}

1. Becker C, Assouad J, Riquet M, et al. Postmastectomy lymphedema: long-term results following microsurgical lymph node transplantation. Ann Surg 2006;243:313-5.

2. Vignes S, Blanchard M, Yannoutsos A, et al. Complications of autologous lymph-node transplantation for limb lymphoedema. Eur J Vasc Endovasc Surg 2013;45:516-20.

3. Mardonado AA, Chen R, Chang DW. The use of supraclavicular free flap with vascularized lymph node transfer for treatment of lymphedema: a prospective study of 100 consecutive cases. J Surg Oncol 2017;115:68-71.

4. Ciudad P, Manrique OJ, Agko M, et al. Ileocecal vascularized lymph node transfer for the treatment of extremity lymphedema: a case report. Microsurgery 2017 May 24 [Epub].https://doi.org/10.1002/micr.30186.

5. Nguyen AT, Suami H, Hanasono MM, et al. Long-term outcomes of the minimally invasive free vascularized omental lymphatic flap for the treatment of lymphedema.J Surg Oncol 2017;115:84-9.

6. Tan PW, Goh T, Nonomura H, et al. Hilar vessels of the submandibular and upper jugular neck lymph nodes: anatomical study for vascularized lymph node transfer to extremity lymphedema. Ann Plast Surg 2016;76:117-23.
7. Poccia I, Lin CY, Cheng MH. Platysma-sparing vascularized submental lymph node flap transfer for extremity lymphedema.J Surg Oncol 2017;115:48-53.

8. Allen RJ Jr, Cheng MH. Lymphedema surgery: patient selection and an overview of surgical techniques. J Surg Oncol 2016;113:923-31.

9. Becker C. Autologous lymph node transfers. J Reconstr Microsurg 2016;32:28-33.

10. Koshima I, Inagawa K, Urushibara K, et al. Supermicrosurgical lymphaticovenular anastomosis for the treatment of lymphedema in the upper extremities. J Reconstr Microsurg 2000;16:437-42.

11. Masia J, Pons G, Nardulli ML. Combined surgical treatment in breast cancer-related lymphedema. J Reconstr Microsurg 2016;32:16-27.

12. Hwang KT, Kim SW, Kim JT, et al. Use of lateral intercostal artery perforator free flaps for resurfacing lower extremities. Ann Plast Surg 2013;71:186-90.

13. Narushima M, Yamamoto T, Yamamoto Y, et al. Lateral intercostal artery perforator-based reversed thoracodorsal artery flap for reconstruction of a chronic radiation ulcer of the lower back wall. Ann Plast Surg 2011;67:352-6.

14. Iida T, Narushima M, Hara H, et al. Supermicrosurgical free sensate intercostal artery perforator flap based on the lateral cutaneous branch for plantar reconstruction. J Plast Reconstr Aesthet Surg 2014;67:995-7.

15. Hakakian CS, Lockhart RA, Kulber DA, et al. Lateral intercostal artery perforator flap in breast reconstruction: a simplified pedicle permits an expanded role. Ann Plast Surg 2016;76 Suppl 3:S184-90. 